\title{
Hematolymphoid Lesions of the Sinonasal Tract
}

\author{
Friederike H. Kreisel ${ }^{1}$
}

Received: 28 August 2015/ Accepted: 9 October 2015/Published online: 1 February 2016

(C) Springer Science+Business Media New York 2016

\begin{abstract}
Hematolymphoid neoplasms of the sinonasal tract are rare and the majority represents non-Hodgkin lymphomas. This review will focus on morphologic, immunophenotypic, and genetic characteristics of the most common types of non-Hodgkin lymphoma, namely diffuse large B cell lymphoma and extranodal natural killer/T-cell lymphoma, nasal type, but also include the discussion of less frequent other hematolymphoid entities, such as extranodal plasmacytomas and Rosai-Dorfman disease.
\end{abstract}

Keywords Sinonasal tract - DLBCL - Extranodal NK/T cell lymphoma · Plasmacytoma $\cdot$ Rosai-Dorfman disease

\section{Review}

Hematolymphoid lesions account for approximately $12-17 \%$ of all malignant neoplasms in the nasal cavity and paranasal sinuses, and in more recent studies represent the second most common sinonasal malignancy after squamous cell neoplasms [1-3]. Overall, hematolymphoid lesions in this location are rare with non-Hodgkin lymphomas, the most common subtype, accounting for $<2 \%$ of all non-Hodgkin lymphomas in Western countries [4]. Together with the Waldeyer's ring and the oral cavity, the sinonasal tract forms the upper aerodigestive tract, and except for the Waldeyer's tonsillar ring, which is considered a nodal site in the Ann Arbor staging system, all other

Friederike H. Kreisel

fkreisel@path.wustl.edu

1 Department of Pathology and Immunology, Washington University, 660 S. Euclid Ave, Box 8118, St. Louis, MO 63110, USA locations within this tract are defined as extranodal sites. The incidence of certain histopathologic types of sinonasal lymphoma differs among populations of different geographic regions. Diffuse large B-cell lymphoma (DLBCL) represents the most common type of lymphoma in the United States and Europe, while extranodal natural killer/ T-cell (NK/T-cell) lymphoma, nasal type, is more prevalent in Asians and the native American population of Mexico, Central America, and South America [3, 5-8].

\section{Diffuse Large B-Cell Lymphoma}

The most common location for DLBCL is the maxillary sinus, followed by the nasal cavity and ethmoid sinus [6, 9]. Patients are usually diagnosed in their sixth decade of life, and due to the relatively non-specific symptoms of rhinorrhea, bloody discharge, facial swelling, or nasal obstruction which can also be associated with benign inflammatory conditions, diagnosis may be delayed. Symptoms of fever, night sweats, and weight loss are not frequent and reported to occur in up to $9 \%$ of patients [3, $8,10]$. Its histology does not differ from any other site of involvement and is characterized by a diffuse growth of large B lymphoid cells with a nuclear size similar or exceeding a normal macrophage nucleus or exceeding twice the size of a normal lymphocyte (Fig. 1). Common morphologic variants include the centroblastic, the immunoblastic, and the anaplastic variant. The centroblastic variant, which is the most common of the three, is characterized by vesicular nuclei with two to four membrane bound nucleoli, in contrast to the immunoblastic variant demonstrating a single, centrally located nucleolus and appreciable amounts of basophilic cytoplasm. The anaplastic variant usually reveals very large bizarre and pleomorphic nuclei reminiscent of Reed-Sternberg cells 

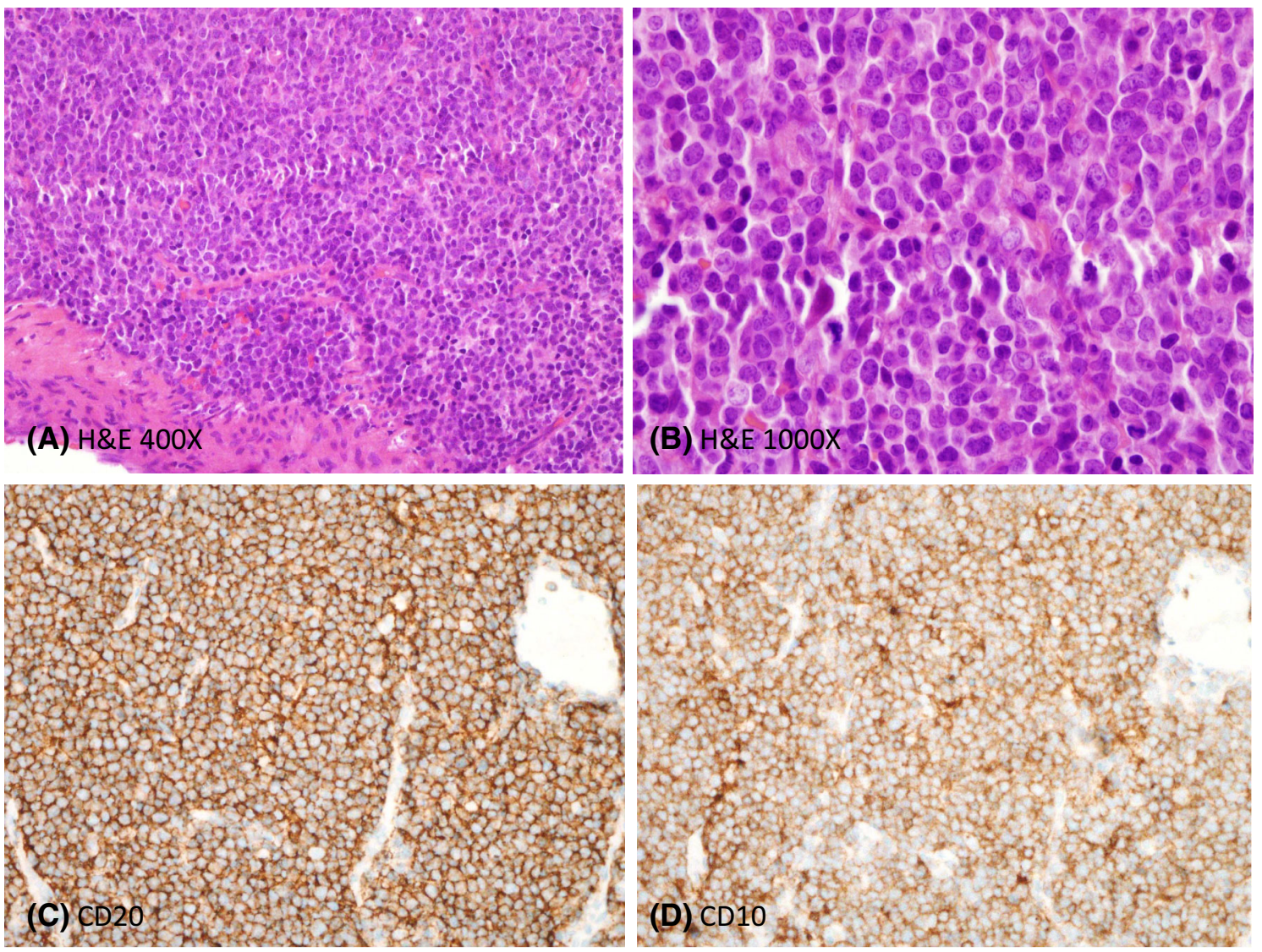

Fig. 1 Diffuse large B-cell lymphoma involving the maxillary sinus. a, b Diffuse sheets of large mature lymphoid cells with irregular nuclei and vesicular chromatin, as well as interspersed mitotic figures (hematoxylin and eosin, $\times 400$ and $\times 1000$ ). $\mathbf{c}$ Neoplastic cells

are uniformly immunoreactive for the B-cell associated marker CD20 $(\times 400)$. d CD10 is uniformly positive, suggesting a germinal center derived subtype of diffuse large B-cell lymphoma $(\times 400)$

and variants, or lymphoma cells of anaplastic large T-cell lymphoma. As with any subtype of B-cell lymphoma, neoplastic cells usually express pan B-cell markers, such as CD19, CD20, CD22, and CD79a (Fig. 1). CD30 may be expressed, especially in the anaplastic variant. CD10, BCL6 , and interferon regulatory factor-4/multiple myeloma- 1 (IRF4/MUM1) expression varies greatly in the literature, and its combination is now increasingly used in the subdivision of the DLBCL into the germinal center like (GCB) and non-germinal center like (non-GCB) subgroup ("Hans classifier") [11]. Cases with CD10 expression in more than $30 \%$ of lymphoma cells fall into the GCB group, as are the cases that are CD10 negative, BCL-6 positive, and IRF4/ MUM1 negative. All other expression patterns are regarded as non-GCB type. However, immunohistochemical assignment into the two prognostic groups does not consistently correlate with the original gene expression profiling performed on untreated, de novo DLBCL, which recognized the GCB-like type to express a gene signature resembling normal germinal center $\mathrm{B}$ cells and to be associated with better event-free and overall survival, and

the non-GCB type or activated B-cell like (ABC-like) type to be closely related with a gene signature reminiscent of activated peripheral blood B-cells and a worse prognosis [12]. Reports are conflicting whether the addition of rituximab to standard chemotherapy abolishes the prognostic value of these two immunohistochemically defined subtypes of DLBCL [13]. A recent registry study of 852 patients with sinonasal DLBCL performed by the Surveillance, Epidemiology and End Results (SEER) program revealed a 5-years disease-specific survival rate of approximately $65 \%$ [9]. Treatment of choice appears to be six cycles of rituximab-cyclophosphamide, doxorubicin, vincristine, and predisone (R-CHOP) [10]. A shorter course of R-CHOP followed by involved field radiotherapy (IFRT) may be beneficial for non-bulky localized DLBCL [10].

\section{Extranodal NK/T-Cell Lymphoma}

Extranodal NK/T-cell lymphoma, nasal type arises predominantly from activated NK cells, although a small percentage can develop from cytotoxic $\mathrm{T}$ lymphocytes. 


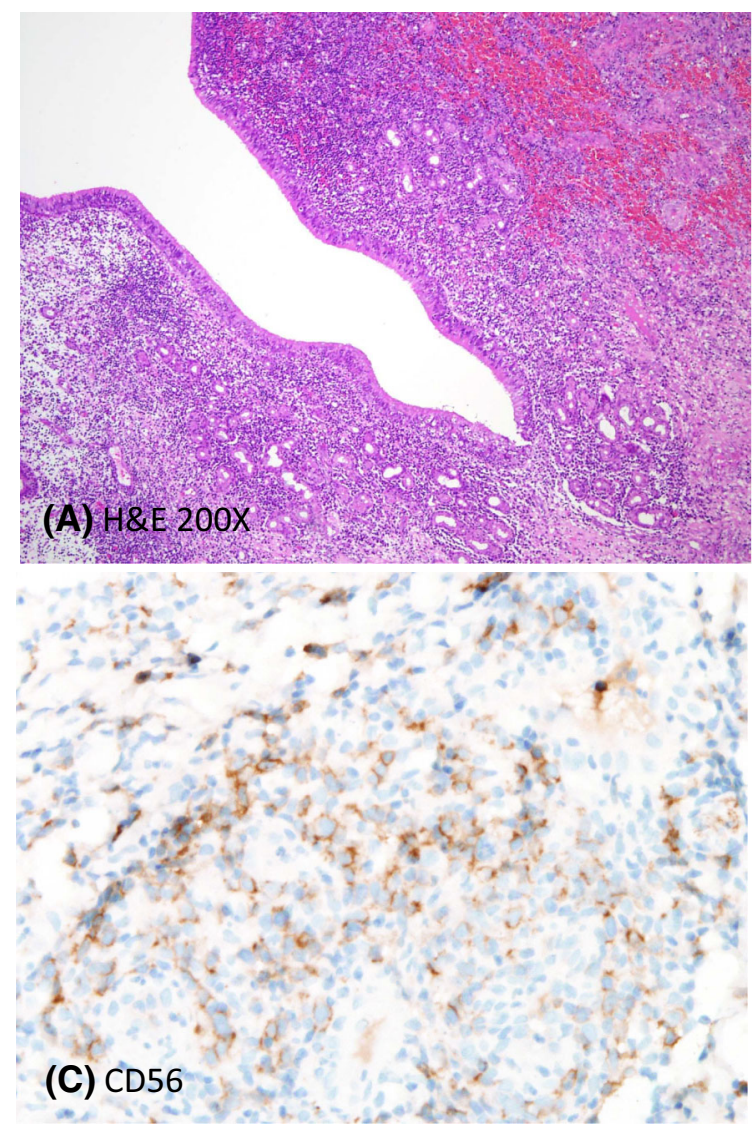

Fig. 2 Extranodal NK/T-cell lymphoma, nasal type. a, b A diffuse infiltrate of medium sized lymphoma cells infiltrating mucosa (a; hematoxylin and eosin, $\times 400)$ and showing angiodestruction (b; hematoxylin and eosin, $\times 1000$ ). Interspersed eosinophils, small lymphocytes, and neutrophils are also present. Neoplastic cells

Compared to sinonasal DLBCL the mean age at diagnosis is lower at approximately 52 years [3]. The nasal cavity represents the prototypic site of involvement [6, 8]. It is formerly known as lethal midline granuloma due to its necrotizing and invasive growth into surrounding bone, cartilage and soft tissue causing severely disfiguring midfacial destructive lesions or angiocentric lymphoma because of its propensity for invading and destroying blood vessels. Although the sinonasal tract is most commonly involved, other preferential sites of extranasal involvement include the skin, soft tissue, gastrointestinal tract, and testis [14]. A defining feature is the association of this lymphoma with the Epstein-Barr virus (EBV) with practically all cases demonstrating EBV encoded RNA (EBER) by in situ hybridization, suggesting a probable pathogenic role of the virus in the development of this lymphoma. Histologically, the lymphomatous infiltrate may exhibit a broad cytologic spectrum with varying proportions of admixed reactive lymphocytes, plasma cells, and histiocytes, imparting the appearance of a benign inflammatory process if neoplastic cells are relatively sparsely represented. Over time,

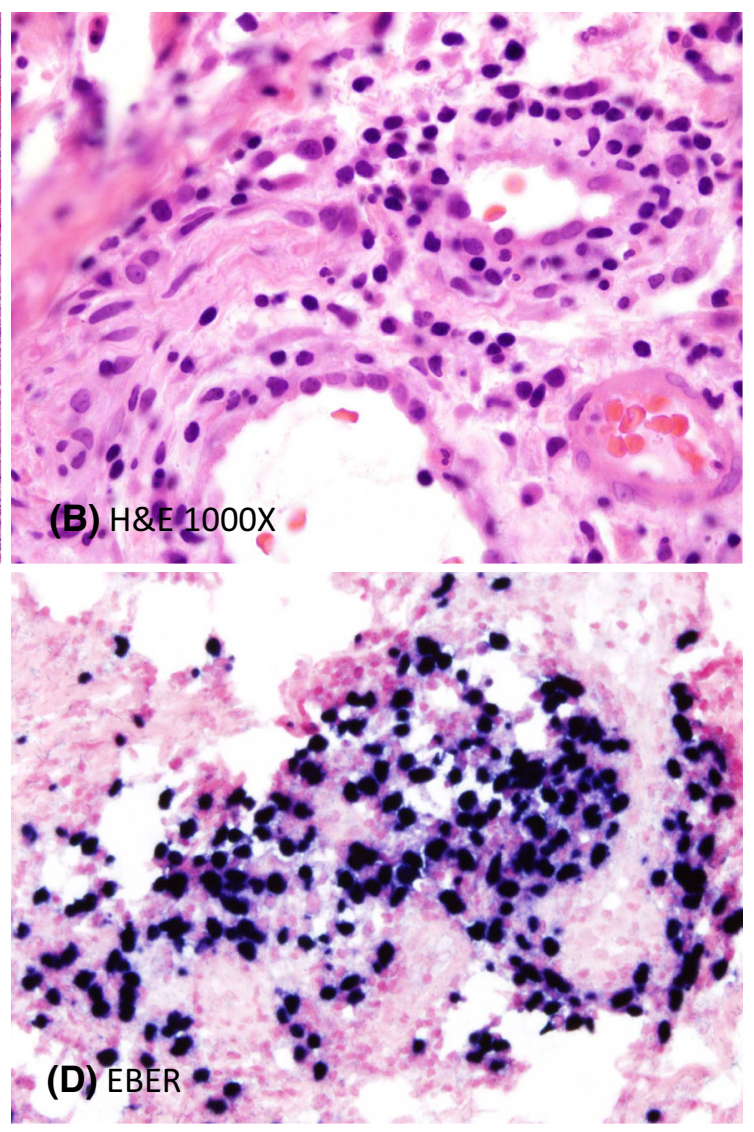

demonstrate irregular, elongated nuclei and hyperchromatic nuclei. c Lymphoma cells weakly co-express CD56 $(\times 400)$. d In situ hybridization for EBER shows strong nuclear staining in neoplastic cells $(\times 400)$

neoplastic cells conglomerate to diffuse sheets causing extensive mucosal ulceration, angiodestruction of blood vessels, and wide spacing of mucosal glands. Interspersed apoptotic bodies, mitotic figures, and coagulative necrosis are common, and hemophagocytosis may be seen (Fig. 2). The size of neoplastic cells can range from small over medium to large, and commonly, nuclei show irregularly folded and angulated, hyperchromatic nuclei with inconspicuous nucleoli (Fig. 2). Lymphoma cells have moderate amounts of cytoplasm, which is often pale to clear. The most typical immunophenotype of extranodal NK/T-cell lymphoma, nasal type is a CD2 positive, CD56 positive, CD43 positive, surface $\mathrm{CD} 3$ negative, but cytoplasmic $\mathrm{CD} 3$ positive profile with co-expression of the cytotoxic molecules granzyme B, T-cell restricted intracellular antigen (TIA-1), and perforin (Fig. 2). Unlike T-cells, NK-cells do not display the complete T-cell receptor complex, but usually express the epsilon chain of $\mathrm{CD} 3$ in the cytoplasm, which can be detected by polyclonal antibodies to $\mathrm{CD} 3$ by immunohistochemistry. Other T-cell or NK-associated antigens, such as CD4, CD5, CD8, CD16, and CD57 are 
Table 1 Clinical, morphologic and immunophenotypic characteristics of sinonasal DLBCL and extranodal NK/T-cell, nasal type

\begin{tabular}{|c|c|c|}
\hline & Diffuse large B-cell lymphoma (DLBCL) & Extranodal NK/T-cell lymphoma, nasal type \\
\hline Age distribution & 6th and 7 th decade & 5th and 6th decade \\
\hline Geographic distribution & Western countries & Asia, Mexico, Central and South America \\
\hline Preferred site of involvement & Maxillary sinus & Nasal cavity \\
\hline Morphologic characteristics & $\begin{array}{l}\text { Sheets of large lymphoid cells, immunoblastic, } \\
\text { centroblastic, or anaplastic in appearance; increased } \\
\text { mitoses, apoptotic debris, or necrosis; } \\
\text { angiodestruction rare }\end{array}$ & $\begin{array}{l}\text { Broad cytologic spectrum with varying proportions of } \\
\text { admixed reactive lymphocytes, plasma cells, and } \\
\text { histiocytes; usually medium-sized lymphoma cells } \\
\text { with irregular, elongated, hyperchromatic nuclei; } \\
\text { frequent mucosal ulceration and angiodestruction; } \\
\text { hemophagocytosis may be present }\end{array}$ \\
\hline Immunophenotype & $\begin{array}{l}\text { Pan-B-cell markers CD19, CD20, CD22, and CD79a; } \\
\text { CD } 43 \text {, CD } 30 \text { expression may be seen; CD10 } \\
\text { positivity in }>30 \% \text { of lymphoma cells suggests } \\
\text { germinal center derived subtype }\end{array}$ & $\begin{array}{l}\mathrm{CD} 2 \text {, CD56, CD43, cytoplasmic CD3 due to epsilon } \\
\text { portion in cytoplasm, cytotoxic granules granzyme B, } \\
\text { perforin, and T-cell restricted intracellular antigen } \\
\text { (TIA-1) }\end{array}$ \\
\hline $\begin{array}{l}\text { Overall 5-years disease- } \\
\text { specific survival based on } \\
\text { the NCI-SEER registry( } 3 \text { ) }\end{array}$ & $63.5 \%$ & $30.9 \%$ \\
\hline
\end{tabular}

NCI-SEER national cancer institute—surveillance, epidemiology, and end results

usually not expressed. Since practically all cases of extranodal NK/T-cell lymphoma, nasal type are EBER positive by in situ hybridization, this procedure allows for detection of lymphoma cells even within a background of extensive secondary inflammation largely excluding the possibility of benign inflammatory processes considered in the differential diagnosis. T-cell receptor and immunoglobulin genes are in germline configuration, and virtually all cases harbor clonal episomal EBV. Combined gene expression profiling and array-based comparative genomic hybridization assays show that lymphoma cells resemble the activated rather than the resting NK cell stage and transcript levels for granzyme $\mathrm{H}$ are distinctively higher than for peripheral T-cell lymphomas [15]. Deletion of $6 \mathrm{q}$ is found in approximately $40-50 \%$ of cases involving deletions of putative tumor suppressor genes such as FOXO3, AIM1, and PRDN1 [16]. The $6 \mathrm{q}$ deletion represents the commonest cytogenetic abnormality in this entity [14]. Overall survival for this type of lymphoma is poor and worse than for sinonasal DLBCL with a 5-years disease specific survival (DSS) of only $31 \%$ compared to approximately $65 \%$ for DLBCL [3]. Clinical, morphologic, and immunophenotypic characteristics of DLBCL and extranodal NK/T-cell lymphoma, nasal type, involving the sinonasal tract are summarized in Table 1.

\section{Extramedullary Plasmacytoma}

Approximately $80 \%$ of solitary extramedullary (extraosseous) plasmacytomas are diagnosed in the upper respiratory tract, with the sinonasal region representing the most common site of involvement ranging from 35 to $54 \%$ [1719]. The diagnosis of an extramedullary plasmacytoma can only be made in the absence of systemic disease. Besides a biopsy proven solitary soft tissue lesion with evidence of clonal plasma cells, it requires the absence of clonal plasma cells in the bone marrow, the absence of end-organ damage produced by clonal plasma cells, and no additional lesions by imaging [20] (Table 2). With this full assessment, approximately $10 \%$ of extramedullary plasmacytomas turn out to represent multiple myeloma [21]. Extramedullary plasmacytomas develop from an expansion of clonal, terminally differentiated, immunoglobulin secreting B-cells that typically secrete a single monoclonal immunoglobulin. The median age at diagnosis is roughly 50-60 years, and males are more commonly affected than females showing a maleto-female ratio of 3.7:1 [17, 22]. Symptoms are generally related to the submucosal tumor mass causing compression and obstruction of local structures leading to nasal obstruction, epistaxis, and facial swelling and pain. The morphologic features are similar to other plasma cell neoplasms and can vary from well-differentiated forms indistinguishable from normal plasma cells to less mature, plasmablastic, or anaplastic forms. Well-differentiated plasmacytomas are characterized by sheets of relatively uniform small plasma cells with abundant cytoplasm and eccentrically located nuclei displaying "clockface" chromatin (Fig. 3). In contrast, less mature plasmacytomas show larger cell size, increased nuclear to cytoplasmic ratio, finely dispersed chromatin, and oftentimes prominent nucleoli. The anaplastic variant reveals bizarrely shaped, pleomorphic nuclei or multinucleation, and may not readily be recognized as a plasma cell neoplasm. Reliable cytologic indicators of neoplasia are nuclear immaturity, prominent nucleoli, and nuclear pleomorphism, since these features do not usually occur in reactive plasma cell conditions. Both reactive and neoplastic plasma cells can contain cytoplasmic inclusions composed of immunoglobulin, including so-called Mott cells with multiple "grape like" cytoplasmic inclusions or plasma 
Table 2 Diagnostic criteria for solitary extramedullary plasmacytoma

Biopsy proven solitary soft tissue lesion with evidence of a clonal plasma cell population

No evidence of clonal plasma cells in the bone marrow

Normal skeletal survey and normal magnetic resonance imaging (MRI) of spine and pelvis

Absence of end-organ damage, such as hypercalcemia, renal insufficiency, anemia, or bone lesions (CRAB) that can be attributed to a clonal plasma cell proliferation
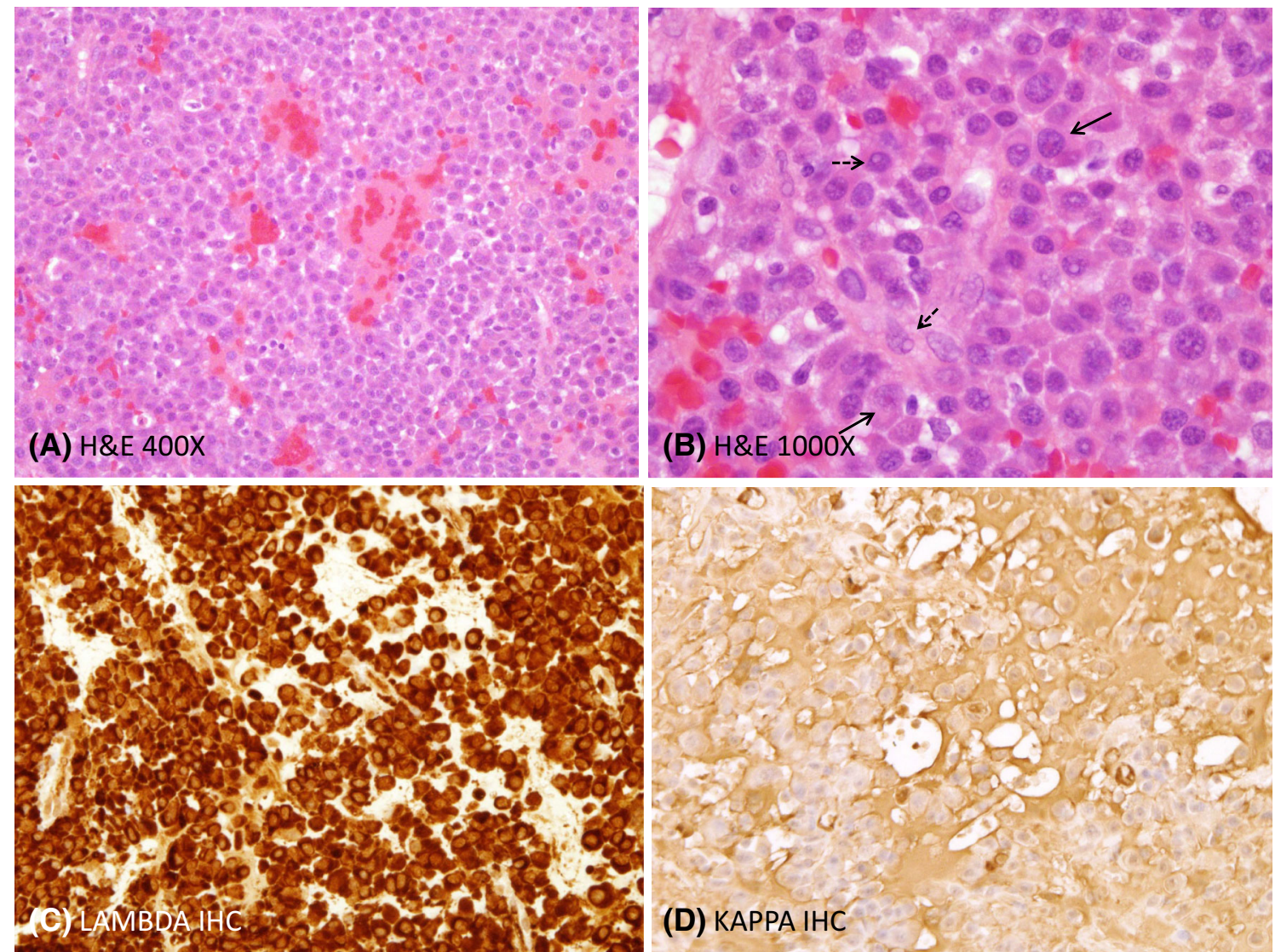

Fig. 3 Extramedullary plasmacytoma involving nasal cavity. a, b Diffuse sheets of relatively small plasma cells with eccentrically located nucleus and abundant eosinophilic cytoplasm (hematoxylin and eosin, $\times 400$ and $\times 1000$ ). Few neoplastic plasma cells show

cells with one large cytoplasmic inclusion with are termed Russel bodies. Plasma cells can also demonstrate cytoplasmic inclusions of immunoglobulin that overlie the nucleus, called Dutcher bodies. These inclusions can be seen in both reactive and neoplastic plasma cells, but are more common in neoplastic conditions. Plasmacytomas typically show their immunoglobulin light chain restriction in the cytoplasm, which can easily be demonstrated by in situ hybridization for light chain mRNA, immunohistochemical staining for light chain antibodies, or by flow cytometry after membrane permeabilization (Figs. 3, 4). Unlike mature normal lymphocytes and most B-cell lymphomas, normal plasma cells or plasma cell neoplasms lack surface immunoglobulin expression. Furthermore, neoplastic plasma cells lose some nucleoli (solid black arrows) or Dutcher bodies (dashed black arrows). c Neoplastic plasma cells stain positively for cytoplasmic lambda light chains $(\times 500)$. d They are negative for kappa light chains $(\times 500)$

of the cell surface antigens related to B-cells, such as CD19. They usually express CD79a, CD138, and strong CD38, similarly to normal plasma cells and may aberrantly co-express CD56, CD117, CD20, CD52, or CD10, in decreasing order of frequency (Fig. 4). Well-differentiated extramedullary plasmacytomas may be difficult to distinguish from marginal zone lymphoma of mucosa-associated lymphoid tissue (MALT) type, especially if plasmacytic differentiation is pronounced and flow cytometry to assess for a related clonal (surface) B-cell population is not available [23]. Uniform expression of several B-cell associated markers such as CD19 (usually lost on malignant plasma cells), CD20, CD79a, and CD22, as well as strong expression of the pan-hematopoietic marker CD45, which is usually 
Fig. 4 Flow cytometry immunophenotyping of aplasma cell neoplasm. Neoplastic plasma cells (orange) are positive for CD38 (a and b) and show cytoplasmiclambda light chain restriction (c).

Furthermore, CD19 is aberrantly lost (d), while CD56 is aberrantly gained (e).
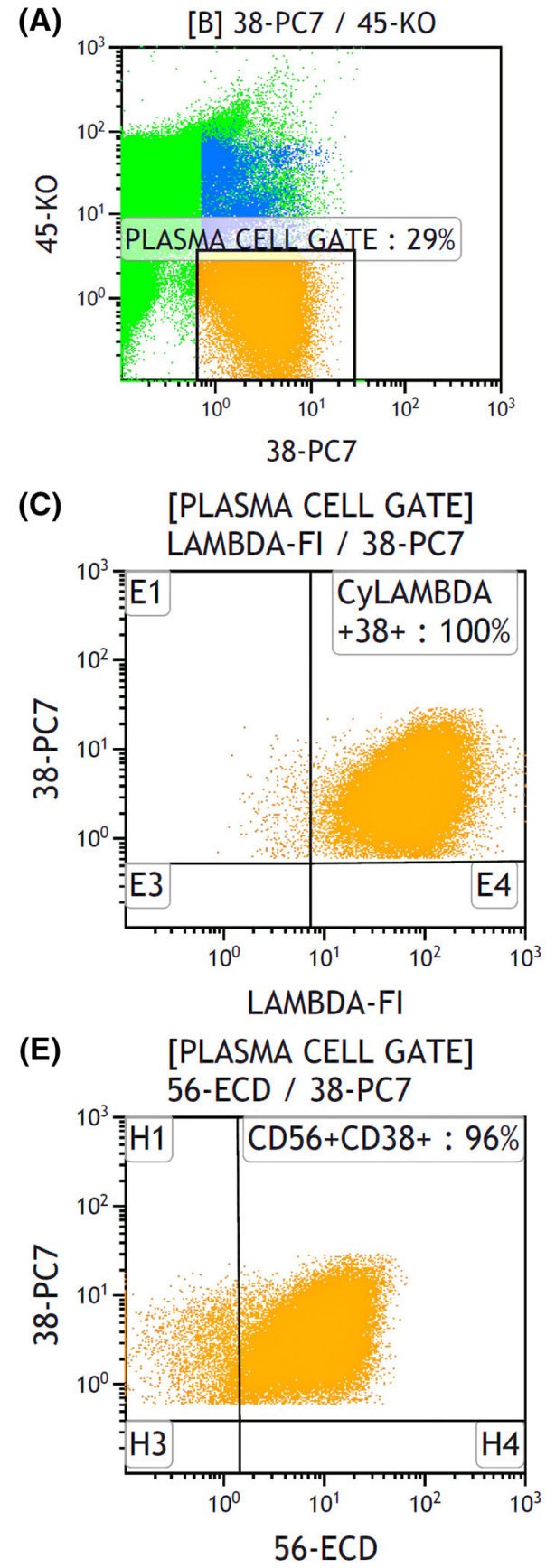
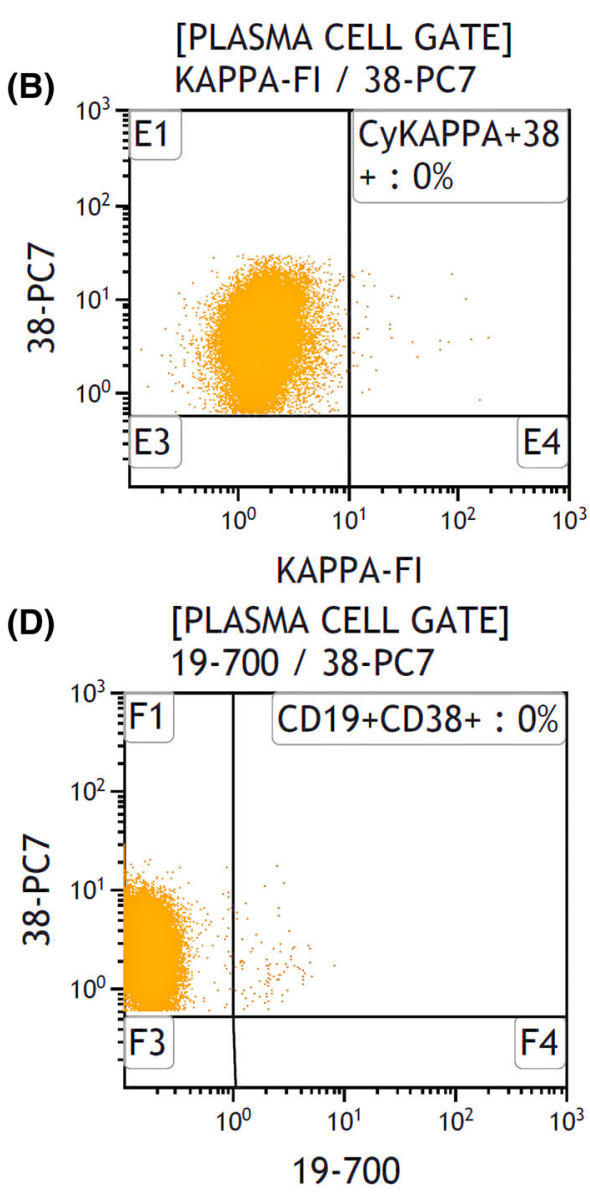

weak to absent on neoplastic plasma cells, favor a marginal zone lymphoma over plasmacytoma. Furthermore, cytogenetics may aide in better delineating these two entities. Chromosomal translocations involving the fibroblast growth factor receptor 3 (FGFR3) and immunoglobulin heavy chain $(I G H)[\mathrm{t}(4 ; 14)(\mathrm{p} 16 ; \mathrm{q} 32)]$, loss of $13 \mathrm{q} 14$ or chromosomal gains involving chromosomes 1, 5, 8, 9, 11 and 15 are seen in extramedullary plasmacytomas, whereas breaks involving MALT1, BCL6, or FOX1 favor a marginal zone lymphoma of
MALT type [24]. Extramedullary plasmacytoma carries a favorable prognosis, which is better than that for solitary plasmacytoma of the bone (solitary osseous plasmacytoma). Progression to multiple myeloma is believed to occur in $48-85 \%$ of osseous plasmacytomas and in only $15-20 \%$ of extramedullary plasmacytomas [17, 19, 25]. The optimal management of extramedullary plasmacytomas is controversial, but the majority of patients can be cured by local radiotherapy [19]. Extramedullary plasmacytomas with 

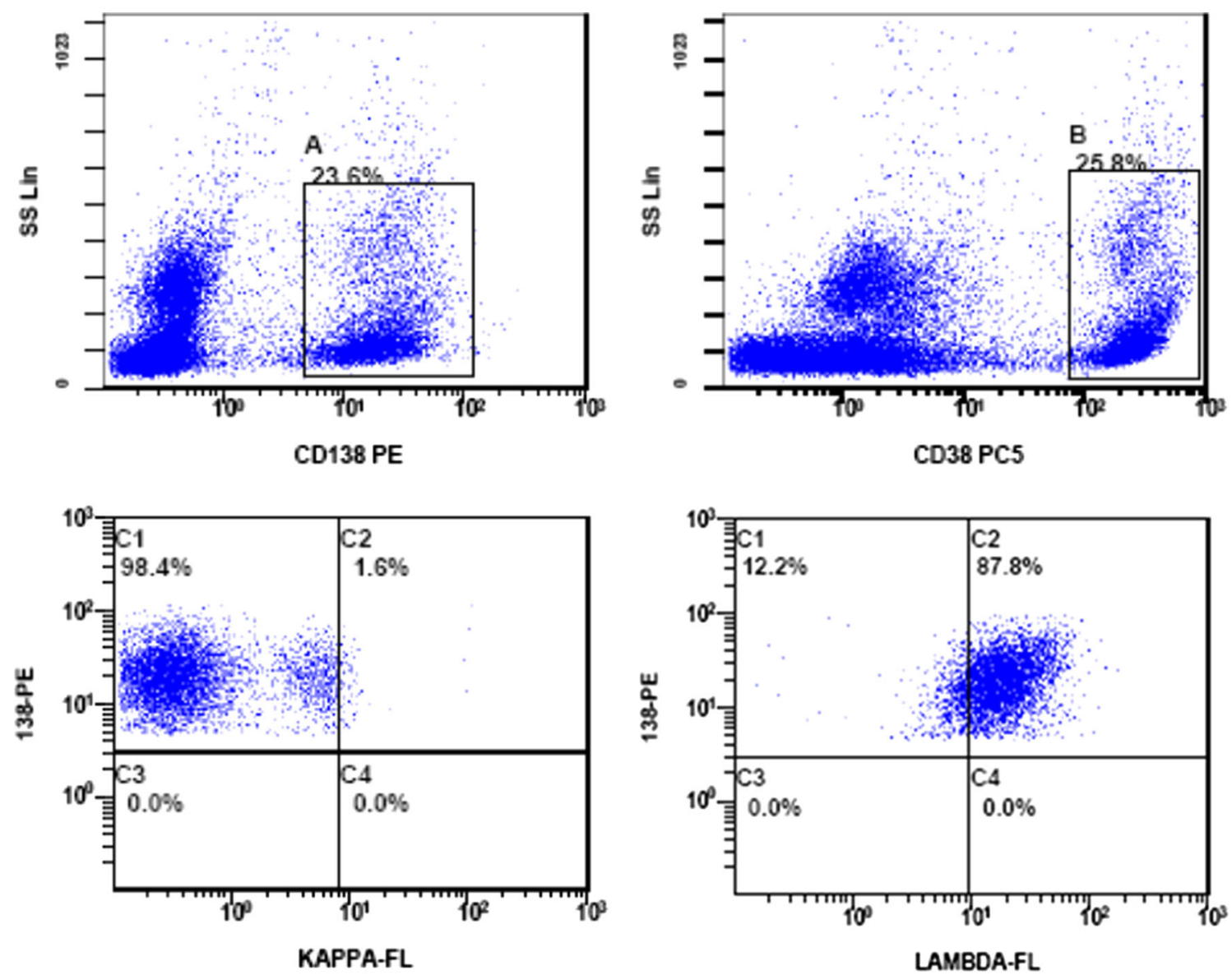

Fig. 5 Extranodal Rosai-Dorfman disease involving head and neck. a, b Aggregates of Rosai-Dorfman histiocytes with pale cytoplasm and surrounding inflammatory cells (a; hematoxylin and eosin,

$\times 200)$. Emperipolesis is present $(\mathbf{b}$; hematoxylin and eosin, $\times 1000)$. c Rosai-Dorfman histiocytes are immunoreactive for CD68 (c) and $\mathrm{S} 100(\mathbf{d})(\times 400)$

plasmablastic morphology have to be distinguished from plasmablastic lymphoma (PBL), which represents a highgrade lymphoma associated with human immunodeficiency virus (HIV) infection, initially described by Delecluse et al. [26] in 1997. Clinically, it is characterized by frequent extranodal occurrence with a particular predilection for the oral cavity [26]. The neoplastic cells in PBL are generally immunoblastic in appearance and show strong expression of plasma cell-associated antigens such as CD38 and CD138 and weak to absent expression of B-cell-associated markers CD19, CD20, and PAX-5 [26, 27]. The high-grade nature of this lymphoma is also represented by numerous interspersed "tingible-body macrophages", imparting a starry-sky appearance and brisk mitotic activity [28]. Although immunohistochemistry for Epstein-Barr virus latent membrane protein 1 (EBV-LMP1) is negative in the majority of cases, in situ hybridization for EBER is usually positive and most useful in differentiating it from a plasmablastic plasmacytoma, which consistently lacks expression of EBER.

\section{Rosai-Dorfman Disease}

Rosai-Dorfman disease (RDD), also known as sinus histiocytosis with massive lymphadenopathy, was first described as a distinct clinicopathologic entity in 1969 by Rosai and Dorfman [29]. It represents a rare non-neoplastic histiocytic proliferation most commonly manifesting in children and young adults as massive painless cervical lymphadenopathy with associated fevers and leukocytosis [30]. Approximately $40 \%$ of cases show extranodal involvement commonly including skin, bone, and head and neck [30]. The most common extranodal sites in the head and neck are the nasal cavity, paranasal sinuses, and orbit [31]. Sinonasal RDD usually presents as a submucosal pseudotumor without bony destruction. Concurrent involvement of lymph nodes or other extranodal sites is common, ranging between 30 and $50 \%$ for lymph nodes and between 40 and $67 \%$ for other extranodal sites [32, 33]. The etiology of RDD is largely unknown, although 
some evidence suggests that immune dysregulation and viral infections, such as caused by human herpesvirus (HHV), parvovirus B19, and Epstein-Barr virus (EBV) may play a role in the pathogenesis [34, 35]. Rosai-Dorfman disease has also been reported in association with immunoglobulin (Ig) G4-related disease [36-38]. However, recent studies are conflicting regarding a true association with IgG4-related disease. Liu et al. [38] report in a study of twenty-nine patients with either nodal or extranodal RDD that the number of IgG4-positive plasma cells and the $\operatorname{IgG} 4 / \mathrm{IgG}$ ratio in these cases, using the strict criteria based on the IgG4-related disease guidelines, was significantly lower than in the ten cases of proven IgG4related disease used as a control group, but similar to results of ten reactive lymph node cases.

Emperipolesis with lymphocytes, or plasma cells, or occasional neutrophils in the histiocyte cytoplasm is the hallmark of Rosai-Dorfman disease. During their transit through the histiocyte cytoplasm, these inflammatory cells remain intact and protected from degradation by cytolytic enzymes. Compared to lymph node lesions, which are characterized by distended sinuses, extranodal RDD usually reveals more fibrosis, decreased numbers of Rosai-Dorfman histiocytes and less pronounced emperipolesis, at times mimicking a fibrohistiocytic tumor or inflammatory pseudotumor (Fig. 5). Immunohistochemically, the Rosai-Dorfman histiocytes characteristically express S100, typically in a nuclear and cytoplasmic pattern. S100 is very helpful in highlighting emperipolesis, since engulfed inflammatory cells are not stained by this marker (Fig. 5d, arrow). The histiocytic markers CD68 and CD163 are positive as well, while CD1a and langerin are negative, excluding a diagnosis of Langerhans cell histiocytosis. Because of its benign and self-limiting nature, treatment of RDD is advised only in patients who have symptoms or if vital organs are affected [30]. Surgery is reserved for diagnostic biopsy or symptomatic relief of compressive lesions. In general, there is no consensus on treatment guidelines for sinonasal RosaiDorfman disease, and treatment usually is assessed on an individual basis.

\section{References}

1. Grau C, Holmelund Jakobsen M, Harbo G, et al. Sino-nasal cancer in Denmark 1982-1991. A nationwide survey. Acta Oncol. 2001;40:19-23.

2. Harbo G, Grau C, Bundgaard T, et al. Cancer of the nasal cavity and paranasal sinuses. A clinic-pathological study of 277 patients. Acat Oncol. 1997;36:45-50.

3. Dubal PM, Dutta R, Vazquez A, et al. A comparative populationbased analysis of sinunasal diffuse large B-cell and extranodal NK/T-cell lymphomas. Laryngoscope. 2015;125:1077-83.
4. Oprea C, Cainap C, Azoulay R, et al. Primary diffuse large B-cell non-Hodgkin lymphoma of the paranasal sinuses: a report of 14 cases. Br J Haematol. 2005;131:468-71.

5. Vega F, Lin P, Medeiros J. Extranodal lymphomas of the head and neck. Ann Diagn Pathol. 2005;9:340-50.

6. Cuadra-Garcia I, Proulx GM, Wu CL, et al. Sinonasal lymphoma: a clinicopathologic analysis of 58 cases from the Massachusetts General Hospital. Am J Surg Pathol. 1999;23:1356-69.

7. Maeshima AM, Taniguchi H, Makita S, et al. Histopathological characteristics of lymphomas in the upper aerodigestive tract: a single-institute study in Japan. J Clin Exp Hematopathol. 2015;55:7-11.

8. Kim GE, Koom WS, Yang W-I, et al. Clinical relevance of three subtypes of primary sinonasal lymphoma characterized by immunophenotypic analysis. Head Neck. 2004;26:584-93.

9. Kanumuri VV, Khan MN, Vazquez A, et al. Diffuse large B-cell lymphoma of the sinonasal tract: analysis of survival in 852 cases. Am J Otolaryngol. 2014;35:154-8.

10. Lee G-W, Go S-I, Kim S-H, et al. Clinical outcome and prognosis of patients with primary sinonasal tract diffuse large B-cell lymphoma treated with rituximab-cyclophosphamide, doxorubicin, vincristine and prednisone chemotherapy: a study by the consortium for improving survival of lymphoma. Leuk Lymphoma. 2015;56:1020-6.

11. Hans CP, Weisenburger DD, Greiner TC, et al. Confirmation of the molecular classification of diffuse large B-cell lymphoma by immunohistochemistry using a tissue microarray. Blood. 2004;103:275-82.

12. Alizadeh AA, Eisen MB, Davis RE, et al. Distinct types of diffuse large B-cell lymphoma identified by gene expression profiling. Nature. 2000;403:503-11.

13. Nyman H, Adde M, Karjalainen-Lindsberg M-L, et al. Prognostic impact of immunohistochemically defined germinal center phenotype in diffuse large B-cell lymphoma patients treated with immunochemotherapy. Blood. 2007;109:4930-5.

14. Chan JKC, Quintanilla-Martinez L, Ferry J, et al. Extranodal NK/ T-cell lymphoma, nasal type. In: Swerdlow SH, Campo E, Harris NL, et al., editors. WHO classification of tumours of haematopoietic and lymphoid tissues. 4th ed. Lyon: IARC Press; 2008. p. 285-93.

15. Huang Y, de Leval L, Gaulard P. Molecular underpinning of extranodal NK/T-cell lymphoma. Best Pract Res Clin Hematol. 2013;26:57-74.

16. Huang Y, De Reyniès A, de Leval L, et al. Gene expression profiling identifies emerging oncogenic pathways operating in extranodal NK/T-cell lymphoma, nasal type. Blood. 2010;115:1226-37.

17. D'Aguillo C, Soni RS, Gordhan C, et al. Sinonasal extramedullary plasmcytoma: a systemic review of 175 patients. Int Forum Allergy Rhinol. 2014;4:156-63.

18. Bachar G, Goldstein D, Brown D, et al. Solitary extramedullary plasmacytoma of the head and neck-long term outcome analysis of 68 cases. Head Neck. 2008;8:1012-9.

19. Sasaki R, Yasuda K, Abe E, et al. Multi-institutional analysis of solitary extramedullary plasmacytomaof the head and neck treated with curative radiotherapy. Int J Radiat Oncol Biol Phys. 2012;82:626-34.

20. Rajkumar SV. Multiple myeloma: 2014 update on diagnosis, riskstratification and management. Am J Hematol. 2014;89:999-1009.

21. Hazarika P, Balakrishnan R, Singh R, et al. Solitary extramedullary plasmacytoma of the sinonasal region. Indian J Otolaryngol Head Neck Surg. 2011;63:S33-5.

22. Patel TD, Vazquez A, Choudhary MM, et al. Sinonasal extramedullary plasmacytoma: a population-based incidence and survival analysis. Int Forum of Allergy Rhinol. 2015 (Epub ahead of print). 
23. Hussong JW, Perkins SL, Schnitzer B, et al. Extramedullary plasmacytoma. A form of marginal zone cell lymphoma? Am J Clin Pathol. 1999;111:111-6.

24. Bink K, Haralambieva E, Kremer M, et al. Primary extramedullary plasmacytoma: similarities with and differences from multiple myeloma revealed by interphase cytogenetics. Hematologica. 2008;93:623-6.

25. Majumdar S, Raghavan U, Jones NS. Solitary plasmacytoma and extramedullary plasmacytoma of the paranasal sinuses and soft palate. J Laryngol Otol. 2002;116:962-5.

26. Delecluse HJ, Anagnostopoulos I, Dallenbach F, et al. Plasmablastic lymphomas of the oral cavity: a new entity associated with the human immunodeficiency virus infection. Blood. 1997;4:1413-20.

27. Dong HY, Scadden DT, de Leval L, et al. Plasmablastic lymphoma in HIV-positive patients. An aggressive Epstein-Barr virus-associated extramedullary plsamacytic neoplasm. Am J Surg Pathol. 2005;29:1633-41.

28. Folk GS, Abbondanzo SL, Childers EL, et al. Plasmablastic lymphoma: a clinicopathologic correlation. Ann Diagn Pathol. 2006;10:608-9.

29. Rosai J, Dorfman RF. Sinus histiocytosis with massive lymphadenopathy. A newly recognized benign clinicopathological entity. Arch Pathol. 1969;87:63-70.

30. Dalia S, Sagatys E, Sokol L, et al. Rosai-Dorfman disease: tumor biology, clinical features, pathology, and treatment. Cancer Control. 2014;21:322-7.
31. Gaitonde S. Multifocal, extranodal sinus histiocytosis with massive lymphadenopathy. Arch Pathol Lab Med. 2007;131:1117-21.

32. Duan H-G, Zheng C-Q, Wang D-H, et al. Extranodal sinonasal Rosai-dorfman disease: a clinical study of 10 cases. Eur Arch Otorhinolaryngol. 2015;272:2313-8.

33. Hsiao CH, Tsai TF, Yang TH, et al. clinicopathologic characteristics of Rosai-Dorfman disease in a medical center in northern Taiwan. J Formos Med Assoc. 2006;105:701-7.

34. Luppi M, Barozzi P, Garber R, et al. Expression of human herpesvirus- 6 antigens in benign and malignant lymphoproliferative diseases. Am J Pathol. 1998;153:815-23.

35. Mehraein Y, Wagner M, Remberger K, et al. Parvovirus B19 detected in Rosai-Dorfman disease in nodal and extranodal manifestations. J Clin Pathol. 2006;59:1320-6.

36. Menon MP, Evbuomwan MO, Rosai J, et al. A subset of RosaiDorfman disease cases exhibit increased IgG4 positive plasma cells: another red herring or a true association with IgG4 related disease? Histopathology. 2014;64:455-9.

37. Zhang X, Hyjek E, Vardiman J. A subset of Rosai-Dorfman disease exhibits features of IgG4-related disease. Am J Clin Pathol. 2013;139:622-32.

38. Liu L, Perry AM, Cao W, et al. Relationship between Rosaidorfman disease and IgG4-related disease. Study of 32 cases. Am J Clin Pathol. 2013;140:395-402. 